\title{
Structure of the Cyclic, Cationic Antimicrobial Peptide (KKWWKF) in Octanol Solution: in silico Approach
}

\author{
Seyed Hassan Mortazavi, Mohammad Reza Bozorgmehr ${ }^{*}$, and Mohammad Momen Heravi
}

Department of Chemistry, Mashhad Branch, Islamic Azad University, Mashhad, Iran

* Corresponding author:

tel: $+98-511-8797022$

email: bozorgmehr@mshdiau.ac.ir

Received: September 1, 2019

Accepted: November 12, 2019

DOI: $10.22146 /$ ijc. 49190

\begin{abstract}
Understanding the mechanism of antimicrobial activity of cyclopeptides helps to design new drugs based on these compounds. A common aspect of the mechanisms provided for the creation of antimicrobial activity of cyclopeptides is their interaction with cell membranes. On the other hand, the octanol/water system is a good mimic of the water/membrane interface. Here, the conformational structure of the very short sequences cationic hexapeptide cyclo (Lys-Lys-Trp-Trp-Lys-Phe) has been studied in different concentrations of octanol by molecular dynamics simulation. The concentration of alcohol in the range of experimental concentrations of octanol was considered. The results obtained from calculating the radial distribution function show that the interaction of the peptide with octanol is a mixed of interactions between charged residues with octanol and the interaction of aromatic residues with octanol. These results are in agreement with experimental observations. Also, Lys5 plays a greater role than Lys1 and Lys 2 in the interactions with octanol.
\end{abstract}

Keywords: cyclopeptide; lipophilic solvents; octanol; membrane

\section{- INTRODUCTION}

Antimicrobial peptides are among the new therapeutic compounds [1]. These compounds are the main components of the immune system against invading pathogens [2]. Structurally, antimicrobial peptides have low molecular masses, usually less than $10 \mathrm{kDa}$. Also, they have amphipathic, cationic nature and different sequences and lengths [3]. For two main reasons, the tendency of research on antimicrobial peptides has increased: First, bacteria are resistant to existing drugs. Second, the development of new antibiotics has been restricted [4]. The range of activity of these peptides is extensive and includes Gram-positive bacteria, Gramnegative bacteria, yeast, viruses, and fungi [5]. Although the activity of antimicrobial peptides is low, however, the resistance of the bacteria against them is less observed [6].

A better understanding of the molecular basis of the mechanism of action of antimicrobial peptides helps to design new drugs based on these peptides. The proposed mechanisms for the antimicrobial function of antimicrobial peptides are divided into two major categories such as membrane degradation and elimination of the intracellular cytoplasmic targets [7]. The common aspect of these mechanisms is the interaction of the antimicrobial peptides with the membrane, whether to degrade the membrane or to pass through it and enter the cell. The interactions of the antimicrobial peptides with the membrane occur through regular adhering and the formation of a cavity in the membrane. Another pathway is irregularly adhering to the membrane and unstable membrane, or a mixture of two methods [7-8]. It has also been noted that antimicrobial peptides bind to the membrane through the interactions of positive charges of arginine or lysine residues with negative charges of the membrane surface. During this interactions, the side chain of aromatic residues orientates and enters the membrane [9]. Maintaining the peptide backbone, the orientation of the side chain of aromatic sequences and the electrostatic interactions of charged residues with membranes are important aspects of these mechanisms. In spite of many studies on the activity of antimicrobial peptides, their mechanism of action is not well understood [10]. For example, by replacing L-amino acid with $\mathrm{D}$-amino acid 
in antimicrobial peptides, in most cases, the activity is not completely eliminated. The fact that it is unlikely about a particular receptor [11]. On the other hand, despite the fact that the membrane surface of most mammals is not charged, positive charged antimicrobial peptides interact with it [12]. The experimental study of the interactions of antimicrobial peptides with membranes has some limitations, for example the complex structure of the membrane, the irregularity of the membrane surface and the alteration of the structure of the membrane when labeled in fluorescence methods [13-14]. Hence, in many cases, molecular dynamics simulations have been used to study the mechanisms of action of antimicrobial peptides [15-17].

Here, the structure of the very short sequences cationic hexapeptide cyclo (Lys-Lys-Trp-Trp-Lys-Phe) (c-AMP) has been studied by molecular dynamics simulation. To investigate the effect of hydrophobic forces on this peptide, molecular dynamics simulation was performed at different concentrations of octanol.

\section{- COMPUTATIONAL DETAILS}

The initial structure of c-AMP was taken from the protein database with the code 1skk [18]. 9 simulation boxes were designed. The peptide was placed in the center of the box. In 8 boxes, the number of octanol molecules were added, thus different concentrations of $5,10,15,20$, $25,30,35$ and $40 \%$ of alcohol were prepared. Each box was assigned as OCTx, $(x=0,5,10,15,20,25,30,35$ and $40 \%)$. The detail information of simulation systems were reported in Table 1.

The octanol molecules were randomly placed in the box. Each simulation box was neutralized with chlorine ions. Gromacs software version 5.1.2 [19] and the Gromos 43a1 force field [20] were used for the calculations. To eliminate bad contacts between atoms, steepest descent algorithm was used to minimize simulation systems. Then extensive equilibrations in NVT and NPT were run for 5 ns. Finally, the configuration of simulated systems were sampled for 500 ns. The V-rescale and Berendsen algorithms were used to control the temperature and pressure of the system components, respectively. For these weakcoupling algorithms, a coupling time of 1.0 ps was considered. The PME algorithm with $1.4 \mathrm{~nm}$ cutoff was used to calculate the electrostatic charge. The chemical bonding of non-solvent components was fixed with the LINCS algorithm [21] and chemical bonding of solvent molecules with SETTLE algorithm [22]. Therefore, the simulation time step was set to 2 fs. To avoid any dependency on the initial conditions all simulations were repeated.

\section{- RESULTS AND DISCUSSION}

The key aspects of mechanism of antimicrobial activity of cyclopeptides are the interaction strength of residues and membrane [9]. Therefore, these criteria were studied in this works. To study the interaction of cAMP residues with solvent in simulated systems, the radial distribution function (RDF) was used. The RDF between particles of type A and B is defined as follow:

$$
\mathrm{g}_{\mathrm{AB}}(\mathrm{r})=\frac{\left\langle\rho_{\mathrm{B}}(\mathrm{r})\right\rangle}{\left\langle\rho_{\mathrm{B}}\right\rangle_{\text {local }}}=\frac{1}{\left\langle\rho_{\mathrm{B}}\right\rangle_{\text {local }}} \frac{1}{\mathrm{~N}_{\mathrm{A}}} \sum_{\mathrm{i} \in \mathrm{A}}^{\mathrm{N}_{\mathrm{A}}} \sum_{\mathrm{j} \in \mathrm{B}}^{\mathrm{N}_{\mathrm{B}}} \frac{\delta\left(\mathrm{r}_{\mathrm{ij}}-\mathrm{r}\right)}{4 \pi \mathrm{r}^{2}}
$$

Table 1. The dimensions of the designed simulation boxes with the number of solvent molecules

\begin{tabular}{lcc}
\hline System & Box dimensions $\left(\mathrm{nm}^{3}\right)$ & Number of water molecules \\
\hline OCT0 & $3.05^{\star} 4.06^{\star} 3.07$ & 1214 \\
OCT5 & $3.04^{\star} 4.04^{\star} 3.06$ & 952 \\
OCT10 & $3.04^{\star} 4.04^{\star} 3.06$ & 701 \\
OCT15 & $3.04^{\star} 4.05^{\star} 3.07$ & 489 \\
OCT20 & $3.11^{\star} 4.05^{\star} 3.07$ & 319 \\
OCT25 & $3.47^{\star} 4.49^{\star} 3.50$ & 546 \\
OCT30 & $3.67^{\star} 4.71^{\star} 3.71$ & 606 \\
OCT35 & $3.85^{\star} 4.85^{\star} 3.87$ & 638 \\
OCT40 & $4.07^{\star} 5.09^{\star} 4.09$ & 819 \\
\hline
\end{tabular}


which $\left\langle\rho_{B}(r)\right\rangle$ is the particle density of type $B$ at a distance $r$ around $A$, and $\left\langle\rho_{B}\right\rangle_{\text {local }}$ is the particle density of type $B$ averaged over all spheres around particles A. The c-AMP peptide has six residues which are lysine, lysine, tryptophan, tryptophan, lysine and phenylalanine, respectively. The c-AMP residues are divided into two groups of charged residues and aromatic residues. The graphs of the RDF of the water's oxygen atoms surrounding the $\mathrm{N}_{\zeta}$ atom of the lysine's side chain are shown in the Fig. 1.
In this figure, the black, red and green colors are denoted for Lys1, Lys2 and Lys5, respectively. According to the Fig. 1, in the absence of alcohol, the height of the first peak in RDF is similar for the three lysine residues. In system OCT0, the height of the first peak of the RDF is slightly increased relative to the height of the first peak of the Lys1's RDF However, the overall shape of the RDF is not changed. In system OCT10, the height of the first peak of the RDF is increased relative to the height of the first peak of the RDF in systems OCT0 and OCT5. The
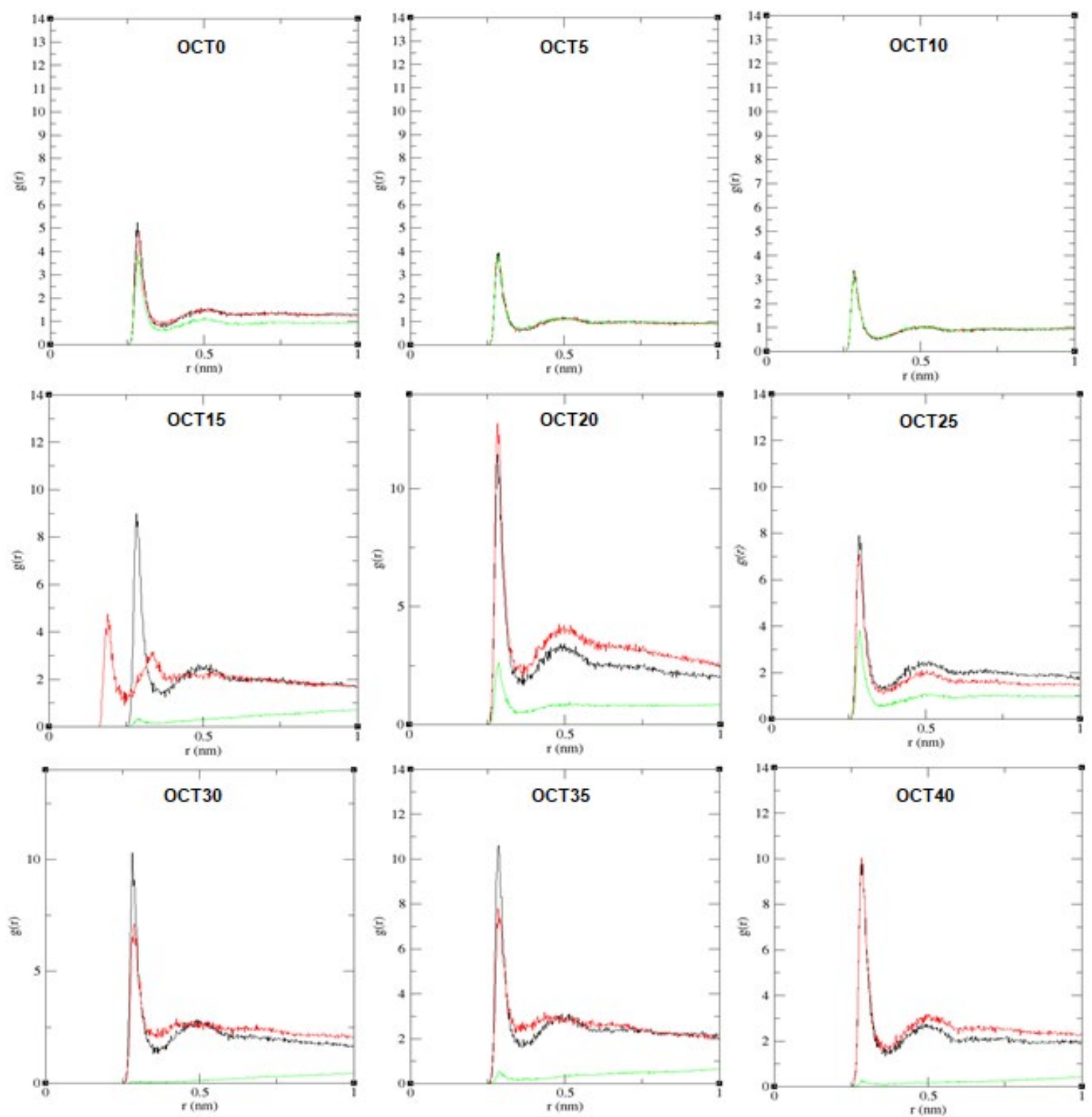

Fig 1. The plots of radial distribution function for $\mathrm{N}_{\zeta}$-Lys.... (water). Black: Lys1, Red: Lys2 and Green: Lys5 
height of the first peak of the RDF for Lys1 and Lys2 increases in system OCT20 and reaches its maximum value in this system. But in the case of Lys5, all of systems with increasing concentration of octanol (OCT25 and OCT40) the height of first peak decreases. Therefore, the interaction of Lys 5 with water is decreased. According to the c-AMP sequence, Lys5 is located between two aromatic residues, while Lys 1 and Lys 2 are bounded by the aromatic residue and charged residue. In Fig. 2, the obtained c-AMP structure from the last simulation step in systems OCT0 and OCT40 is shown.

As can be seen from Fig. 2, the side chain of Lys 5 in the system OCT40 is oriented upward, approximately perpendicular to the peptide ring axis and surrounded by the side chain of Trp4 and Phe6. However, in OCT0 system, the side chain of Lys5 is oriented toward the bottom and is somewhat distant from the side chain of Trp4 and Phe6. The graph of the RDF of the water's oxygen atoms surrounding the $\mathrm{C}_{\zeta}$ atom of the phenylalanine's side chain and the $C_{\eta}$ atom of the tryptophan's side chain is shown in the Fig. 3.

Atoms $\mathrm{C}_{\zeta}$ and $\mathrm{C}_{\eta}$ are the outermost atoms in the side chain of phenylalanine and tryptophan, respectively. Compared to Fig. 1, it is observed that the height of the peak in the RDF is considerably lower. This means that the intensity of the interaction of the aromatic residues with water is greater than the intensity of interactions of the charged residues with water. It is also observed that changes in the interaction of aromatic residues with water molecules due to an increase in the concentration of octanol. The changes in the interaction of charged residues with water molecules due to the increase in concentration of octanol is much lower. This finding agrees with experimental data that indicates that the interaction of charged residues with membranes is greater [8,23-24]. With respect to Fig. 3, it is observed that the Phe6 behavior has changed in 25\% concentration of octanol and its interaction with water molecules is more than that of $\operatorname{Trp} 3$ and Trp4. Therefore, according to Fig. 1 and 3, the orientations of the aromatic residues change the behavior of the charged residues and affects their interactions with the octanol. It is also observed that the effect of alcohol on aromatic residues varies in different concentrations. At higher concentrations of octanol, Trp4 is more affected. This is consistent with the experimental data reported by Dathe et al. [25].

In order to elaborate more detail on other structural aspects, the coordination numbers on c-AMP residues were calculated by integrating the radial distribution function according to the relation $(r)=4 \pi \rho \int_{0}^{r} g(r) r^{2} d r$. In this relation, $g(r)$ is a radial distribution function. The respective coordination number for $\mathrm{N}_{\zeta}$-Lys, $\mathrm{C}_{\zeta}-$ Phe and $\mathrm{C}_{\eta}$-Trp are presented in Table 2.

Based on Table 2, it is seen that the coordination number around the carbon atom of aromatic residues decreases with increasing concentration in most cases. A peculiar behavior is observed for of the nitrogen atom of lysine. These results are in agreement with the results obtained in similar simulations [26].

In Fig. 4, the calculated RMSF values for c-AMP residues in simulated systems have been reported. RMSF is a measure of the flexibility of the peptide residues [2728]. In Fig. 4, for a better comparison of the effect of alcohol, the difference between the amounts of RMSF in

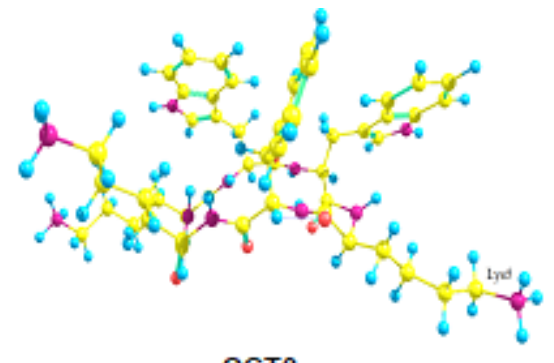

Ост0

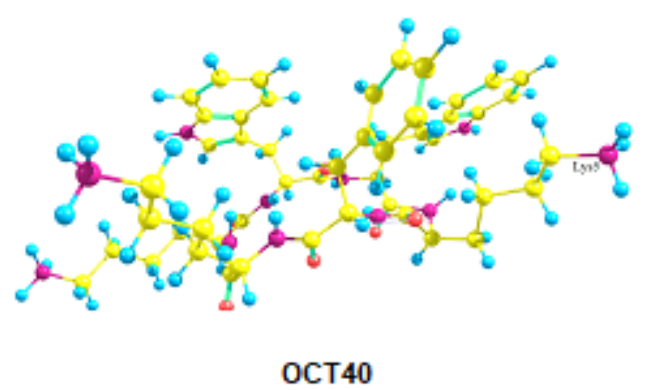

Fig 2. The c-AMP structure obtained from the last simulation step in systems OCT0 and OCT40. Water and octanol molecules eliminate for clarity 

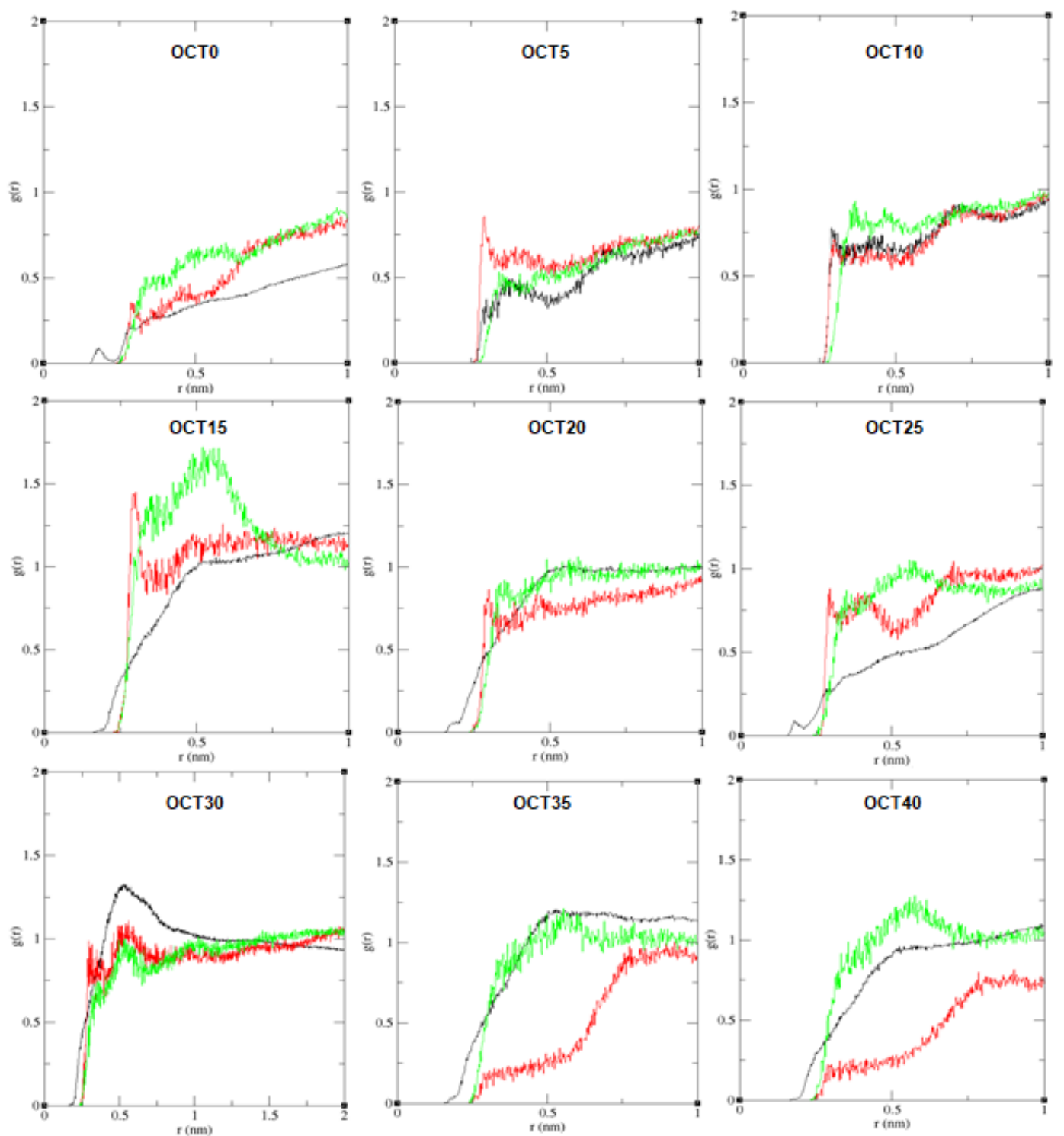

Fig 3. The plots of radial distribution function for $\mathrm{C}_{\zeta}-\mathrm{Phe} \ldots . \mathrm{O}$ (water) and $\mathrm{C}_{\eta}-\operatorname{Trp} \ldots . \mathrm{O}$ (water). Black: Trp3, Red: Trp4 and Green: Phe6

Table 2. The number of water molecules around atoms $\mathrm{N}_{\zeta}-\mathrm{Lys}, \mathrm{C}_{\zeta}-\mathrm{Phe}$ and $\mathrm{C}_{\eta}-\mathrm{Trp}$

\begin{tabular}{llllllllll}
\hline & OCT0 & OCT5 & OCT10 & OCT15 & OCT20 & OCT25 & OCT30 & OCT35 & OCT40 \\
\hline Lys1 & 3.041 & 3.035 & 4.034 & 3.052 & 5.038 & 3.048 & 4.062 & 3.039 & 5.046 \\
Lys2 & 2.055 & 3.061 & 4.053 & 2.049 & 3.062 & 4.052 & 3.066 & 3.048 & 5.039 \\
Trp3 & 2.035 & 3.037 & 4.039 & 5.004 & 5.146 & 5.547 & 5.749 & 5.850 & 6.002 \\
Trp4 & 2.037 & 3.038 & 3.541 & 3.944 & 4.046 & 4.748 & 5.149 & 5.552 & 5.957 \\
Lys5 & 3.146 & 3.642 & 3.951 & 4.355 & 3.762 & 3.448 & 4.049 & 4.050 & 5.156 \\
Phe6 & 3.036 & 3.441 & 3.946 & 4.449 & 4.555 & 4.959 & 5.166 & 5.367 & 5.770 \\
\hline
\end{tabular}

the presence and/or absence of octanol. Regarding Fig. 4, it is observed that the flexibility of the residues is not much affected by octanol.
According to Fig. 4, the range of RMSF changes is about $0.15 \mathrm{~nm}$. In fact, this change value for a peptide with 6 residues is not high. In other words, the backbone 


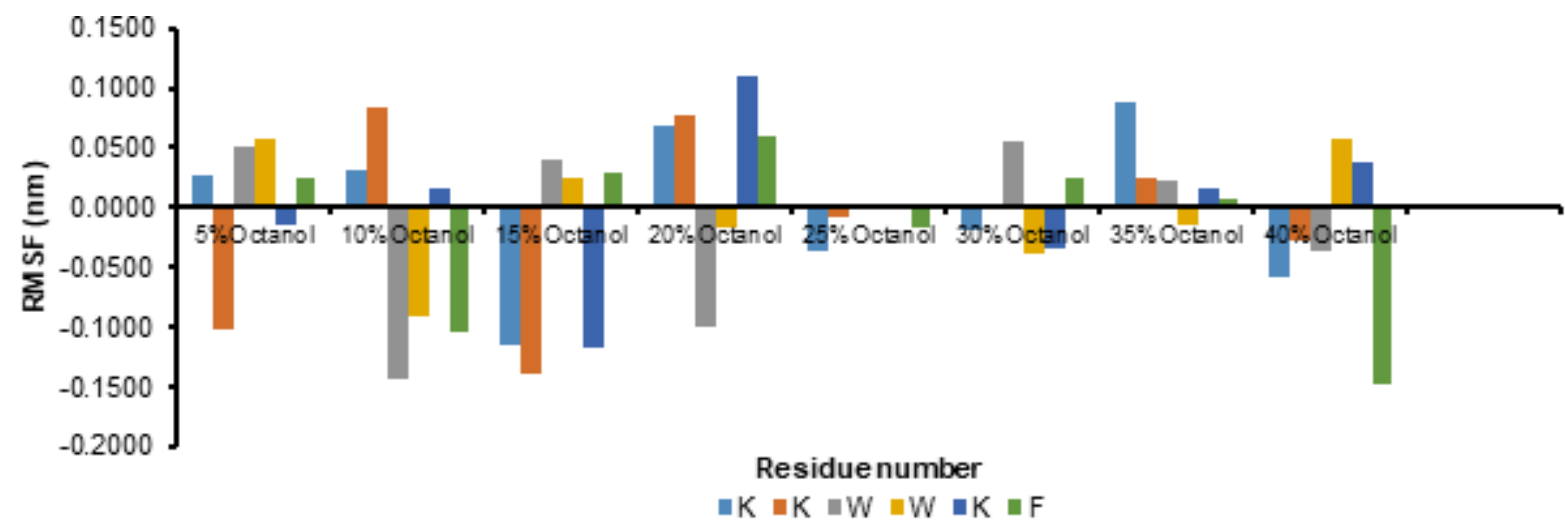

Fig 4. The values of the RMSF difference curve of the c-AMP residues in the systems with the octanol and in the absence of the octanol

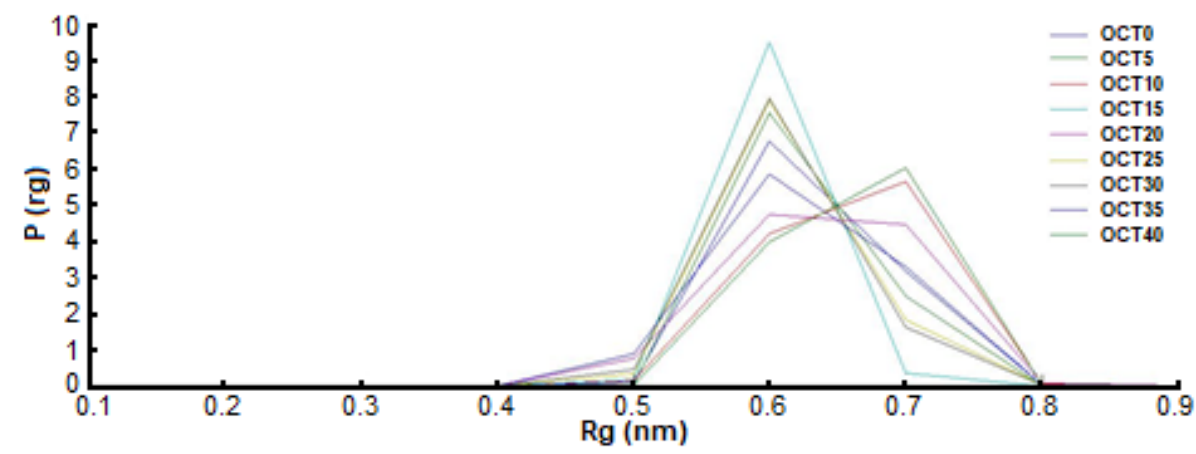

Fig 5. The probability distribution of the radius of gyration in different simulation systems

of c-AMP is maintained when interacting with octanol. This is consistent with experimental evidence that the peptide backbone should be maintained in the interaction of the cyclopeptide with the membrane to preserve the antimicrobial activity [9,29]. A remarkable point in Fig. 4 is at $25 \%$ octanol, the RMSF shows the smallest values. This is consistent with the findings of the radial distribution function in Fig. 1 and 3. To examine the overall shape of the c-AMP, the distribution of the peptide radius of gyration was calculated in various simulated systems. The results are shown in Fig. 5.

Based on Fig. 5, the amplitude of the change in the radius of gyration is about $0.1 \mathrm{~nm}$, thus the presence of alcohol does not affect the overall shape of the peptide. Finally, to investigate the effect of alcohol on peptide dynamics, the respective diffusion coefficients in different systems was calculated. The diffusion coefficient is a criterion of motion of a molecule in a solvent. As the diffusion coefficient value increases, the mobility of the molecule increases [30]. Einstein relation was used to
Table 3. Calculated values of Amyloid $\beta$-peptide (1-42) diffusion coefficient

\begin{tabular}{lc}
\hline System & Diffusion coefficient $\left(\mathrm{D}^{*} 10^{-5} \mathrm{~cm}^{2} / \mathrm{s}\right)$ \\
\hline OCT0 & $0.1473( \pm 0.0830)$ \\
OCT5 & $0.1180( \pm 0.1779)$ \\
OCT10 & $0.0466( \pm 0.0749)$ \\
OCT15 & $0.0515( \pm 0.0467)$ \\
OCT20 & $0.0255( \pm 0.0142)$ \\
OCT25 & $0.0168( \pm 0.0321)$ \\
OCT30 & $0.0013( \pm 0.0147)$ \\
OCT35 & $0.0007( \pm 0.0004)$ \\
OCT40 & $0.0003( \pm 0.0001)$ \\
\hline
\end{tabular}

calculate the diffusion coefficient of c-AMP in all simulated systems:

$\mathrm{D}=\frac{1}{6} \lim _{\mathrm{t} \rightarrow \infty} \frac{\mathrm{d}}{\mathrm{dt}}\left\langle\left|\mathrm{r}_{\mathrm{i}}(\mathrm{t})-\mathrm{r}_{\mathrm{i}}(0)\right|^{2}\right\rangle$

where $r_{i}$ is the atom coordinate vector and the term inside the angle brackets is the mean square displacement (MSD). In this approach, the self-diffusion coefficient (D) is proportional to the slope of the MSD as a function of time in the diffusional regime [31]. The 
diffusion coefficient values of c-AMP listed in Table 3.

Based on Table 3, it can be concluded that increasing alcohol concentration will reduce the mobility of the peptide.

\section{- CONCLUSION}

The structure of cationic hexapeptide cyclo (LysLys-Trp-Trp-Lys-Phe) (c-AMP) was investigated in the presence of different concentrations of octanol. The cAMP shows conformational stability in the presence of octanol. Also, the obtained results show that the interaction of c-AMP with octanol from the beginning occurs through charged residues and then through aromatic residues. These findings results are in good agreement with experimental results. The results obtained from the radial distribution function indicate that the water molecules around the lysine residues are more structurally than in the aromatic residues.

\section{- REFERENCES}

[1] Hancock, R.E., and Sahl, H.G., 2006, Antimicrobial and host-defense peptides as new anti-infective therapeutic strategies, Nat. Biotechnol., 24 (12), 1551-1557.

[2] Reddy, K.V.R., Yedery, R.D., and Aranha, C., 2004, Antimicrobial peptides: Premises and promises, Int. J. Antimicrob. Agents, 24 (6), 536-547.

[3] Yount, N.Y., and Yeaman, M.R., 2004, Multidimensional signatures in antimicrobial peptides, Proc. Natl. Acad. Sci. U.S.A., 101 (19), 7363-7368.

[4] Appelt, C., Schrey, A.K., Söderhäll, J.A., and Schmieder, P., 2007, Design of antimicrobial compounds based on peptide structures, Bioorg. Med. Chem. Lett., 17 (8), 2334-2337.

[5] Marr, A.K., Gooderham, W.J., and Hancock, R.E., 2006, Antibacterial peptides for therapeutic use: Obstacles and realistic outlook, Curr. Opin. Pharmacol., 6 (5), 468-472.

[6] Zasloff, M., 2002, Antimicrobial peptides of multicellular organisms, Nature, 415 (6870), 389-395.

[7] Fjell, C.D., Hiss, J.A., Hancock, R.E., and Schneider, G., 2012, Designing antimicrobial peptides: Form follows function, Nat. Rev. Drug Discovery, 16 (11), 37-51.

[8] Finger, S., Kerth, A., Dathe, M., and Blume, A., 2015, The efficacy of trivalent cyclic hexapeptides to induce lipid clustering in PG/PE membranes correlates with their antimicrobial activity, Biochim. Biophys. Acta, Biomembr., 1848 (11), 2998-3006.

[9] Chan, D.I., Prenner, E.J., and Vogel, H.J., 2006, Tryptophan- and arginine-rich antimicrobial peptides: Structures and mechanisms of action, Biochim. Biophys. Acta, Biomembr., 1758 (9), 11841202.

[10] Hale, J.D., and Hancock, R.E., 2007, Alternative mechanisms of action of cationic antimicrobial peptides on bacteria, Expert Rev. Anti Infect. Ther., 5 (6), 951-959.

[11] Wessolowski, A., Bienert, M., and Dathe, M., 2004, Antimicrobial activity of arginine-and tryptophanrich hexapeptides: The effects of aromatic clusters, D-amino acid substitution and cyclization, J. Pept. Res., 64 (4), 159-169.

[12] Lai, Y., and Gallo, R.L., 2009, AMPed up immunity: how antimicrobial peptides have multiple roles in immune defense, Trends Immunol., 30 (3), 131-141.

[13] Shai, Y., 1999, Mechanism of the binding, insertion and destabilization of phospholipid bilayer membranes by $\alpha$-helical antimicrobial and cell nonselective membrane-lytic peptides, Biochim. Biophys. Acta, Biomembr., 1462 (1-2), 55-70.

[14] Marchal, D., Boireau, W., Laval, J.M., Moiroux, J., and Bourdillon, C., 1998, Electrochemical measurement of lateral diffusion coefficients of ubiquinones and plastoquinones of various isoprenoid chain lengths incorporated in model bilayers, Biophys. J., 74 (4), 1937-1948.

[15] Shepherd, C.M., Vogel, H.J., and Tieleman, D.P., 2003, Interactions of the designed antimicrobial peptide MB21 and truncated dermaseptin S3 with lipid bilayers: Molecular-dynamics simulations, Biochem. J., 370 (1), 233-243.

[16] Jean-François, F., Elezgaray, J., Berson, P., Vacher, P., and Dufourc, E.J., 2008, Pore formation induced 
by an antimicrobial peptide: Electrostatic effects, Biophys. J., 95 (12), 5748-5756.

[17] Wang, Y., Schlamadinger, D.E., Kim, J.E., and McCammon, J.A., 2012, Comparative molecular dynamics simulations of the antimicrobial peptide CM15 in model lipid bilayers, Biochim. Biophys. Acta, Biomembr., 1818 (5), 1402-1409.

[18] Appelt, C., Wessolowski, A., Söderhäll, J.A., Dathe, M., and Schmieder, P., 2005, Structure of the antimicrobial, cationic hexapeptide cyclo (RRWWRF) and its analogues in solution and bound to detergent micelles, ChemBioChem, 6 (9), 1654-1662.

[19] Abraham, M.J., van der Spoel, D., Lindahl, E., Hess, B., and the GROMACS development team, 2016, GROMACS User Manual version 5.1.2, www.gromacs.org.

[20] van Gunsteren, W.F., Billeter, S., Eising, A.A., Hunenberger, P.H., Krüger, P., Mark, A.E., Scott, W.R.P., and Tironi, I.G., 1996, Biomolecular simulation: The GROMOS96 manual and user guide, Hochschulverlag AG an der ETH Zürich, Zürich, Switzerland.

[21] Hess, B., Bekker, H., Berendsen, H.J.C., and Fraaije, J.G.E.M., 1997, LINCS: A linear constraint solver for molecular simulations, J. Comput. Chem., 18 (12), 1463-1472.

[22] Miyamoto, S., and Kollman, P.A., 1992, Settle: An analytical version of the SHAKE and RATTLE algorithm for rigid water models, J. Comput. Chem., 13 (8), 952-962.

[23] Arouri, A., Dathe, M., and Blume, A., 2009, Peptide induced demixing in $\mathrm{PG} / \mathrm{PE}$ lipid mixtures: A mechanism for the specificity of antimicrobial peptides towards bacterial membranes?, Biochim. Biophys. Acta, Biomembr., 1788 (3), 650-659.
[24] Wadhwani, P., Epand, R.F., Heidenreich, N., Bürck, J., Ulrich, A.S., and Epand, R.M., 2012, Membraneactive peptides and the clustering of anionic lipids, Biophys. J., 103 (2), 265-274.

[25] Dathe, M., Nikolenko, H., Klose, J., and Bienert, M., 2004, Cyclization increases the antimicrobial activity and selectivity of arginine-and tryptophancontaining hexapeptides, Biochemistry, 43 (28), 9140-9150.

[26] Jahanbin, F., Bozorgmehr, M.R., Morsali, A., and Beyramabadi, S.A., 2019, The effect of different alcohols on the Asp23-Lys28 and Asp23-Ala42 salt bridges of the most effective peptide in Alzheimer's disease: Molecular dynamics viewpoints, J. Mol. Graphics Modell., 86, 199-208.

[27] Bozorgmehr, M.R., and Monhemi, H., 2015, How can a free amino acid stabilize a protein? Insights from molecular dynamics simulation, J. Solution Chem., 44 (1), 45-53.

[28] Housaindokht, M.R., Bozorgmehr, M.R., and Monhemi, H., 2012, Structural behavior of Candida antarctica lipase $B$ in water and supercritical carbon dioxide: A molecular dynamic simulation study, $J$. Supercrit. Fluids, 63, 180-186.

[29] Almeida, P.F., and Pokorny, A., 2009, Mechanisms of antimicrobial, cytolytic, and cell-penetrating peptides: From kinetics to thermodynamics, Biochemistry, 48 (34), 8083-8093.

[30] Brune, D., and Kim, S., 1993, Predicting protein diffusion coefficients, Proc. Natl. Acad. Sci. U.S.A., 90 (9), 3835-3839.

[31] Frenkel, D., and Smit, B., 2002, Understanding Molecular Simulation: From Algorithms to Applications, $2^{\text {nd }}$ Ed., Academic Press, San Diego, CA. 